\title{
Impact of physician income source on productivity
}

\author{
Mark Otto Baerlocher MD' \\ Jason Noble MD² \\ Allan S Detsky MD, PhD, FRCPC 3
}

University of Toronto Radiology ${ }^{1}$ and Ophthalmology $^{2}$ Residency Programs, Departments of Health Policy Management and Evaluation and Medicine ${ }^{3}$, University of Toronto, and Departments of Medicine ${ }^{3}$, Mount Sinai Hospital and University Health Network

Toronto, Ontario

Manuscript submitted 18th December, 2006

Manuscript accepted 2nd January, 2007

Clin Invest Med 2007; 30 (1): 42-43.
Based on data from the 2004 National Physician Survey $^{1}$, physicians whose primary payment method was fee-for-service saw more patients per week than physicians remunerated by other methods, including salary or blended payments. This result did not change when examined according to specialty or specialty grouping (Table 1), physician age (Table 2) Family physicians versus specialists, type of practice (officebased versus hospital-based; data not shown), or practice setting (urban versus rural; data not shown). Overall, fee-for-service (FFS) physicians saw approximately twice the number of patients per week as salaried physicians. These data provide convincing evidence that FFS physicians see substantially more patients.

There are several possible explanations for this result. However, given the increasing proportion of younger physicians who are being remunerated by sources other than FFS, a decreasing interest among younger physicians in FFS method of payment, and the results presented here demonstrating such a striking difference in the number of patients seen by each group, this issue should be examined in detail, as it will likely have considerable implications with respect to future physician supply. As large segments of the physician workforce change from FFS to non-FFS positions, it is possible the 'extra' patients may simply be seen by those remaining FFS, ultimately driving the costs of health care higher.

\section{References}

1. National Physician Survey 2004 http://www.cfpc.ca/English/cfpc/research/janus\%20pr oject/nps/results/default.asp?s $=1$

Correspondence to:

Mark Otto Baerlocher, MD

Radiology Residency Training Program

University of Toronto, Toronto, Ontario

mark.baerlocher@utoronto.ca 
TABLE 1. Influence of specialty: Patient visits per week (mean \pm SD) and income source.

\begin{tabular}{|c|c|c|c|c|c|}
\hline & $\geq 90 \%$ FFS & $\geq 90 \%$ Contract & $\geq 90 \%$ Sessional & $\geq 90 \%$ Salary & Not $\geq 90 \%$ of 1 method \\
\hline $\begin{array}{l}\text { Ob/Gyn } \\
\text { (n) }\end{array}$ & $\begin{array}{c}108.9 \pm 51.2 \\
(306)\end{array}$ & & & $\begin{array}{c}48.5 \pm 31.9 \\
(32) \\
\end{array}$ & $\begin{array}{c}70.7 \pm 36.5 \\
(122) \\
\end{array}$ \\
\hline $\begin{array}{l}\text { Pediatrics } \\
\text { (n) }\end{array}$ & $\begin{array}{c}109.3 \pm 62.6 \\
(304)\end{array}$ & & & $\begin{array}{c}41.7 \pm 41.8 \\
(118) \\
\end{array}$ & $\begin{array}{c}65.5 \pm 50.9 \\
(288) \\
\end{array}$ \\
\hline $\begin{array}{l}\text { Psychiatry } \\
\text { (n) }\end{array}$ & $\begin{array}{c}45.4 \pm 26.8 \\
(513) \\
\end{array}$ & & $\begin{array}{c}28.9 \pm 20.1 \\
(56) \\
\end{array}$ & $\begin{array}{c}29.2 \pm 16.5 \\
(58) \\
\end{array}$ & $\begin{array}{c}39.4 \pm 23.4 \\
(686) \\
\end{array}$ \\
\hline $\begin{array}{l}\text { General Internal Medicine } \\
\text { (n) }\end{array}$ & $\begin{array}{c}74.7 \pm 50.0 \\
(383)\end{array}$ & & & $\begin{array}{c}44.7 \pm 40.5 \\
(49)\end{array}$ & $\begin{array}{c}60.2 \pm 46.7 \\
(207)\end{array}$ \\
\hline $\begin{array}{l}\text { Medical sub-specialty* } \\
\text { (n) }\end{array}$ & $\begin{array}{c}76.6 \pm 41.6 \\
(708) \\
\end{array}$ & $\begin{array}{c}37.5 \pm 19.8 \\
(36) \\
\end{array}$ & & $\begin{array}{c}40.8 \pm 35.5 \\
(154) \\
\end{array}$ & $\begin{array}{c}55.2 \pm 38.8 \\
(471) \\
\end{array}$ \\
\hline $\begin{array}{l}\text { Surgery** } \\
\text { (n) }\end{array}$ & $\begin{array}{c}100.6 \pm 56.2 \\
(1688) \\
\end{array}$ & $\begin{array}{c}45.9 \pm 29.3 \\
(43) \\
\end{array}$ & & $\begin{array}{c}55.4 \pm 35.9 \\
(99) \\
\end{array}$ & $\begin{array}{c}73.3 \pm 48.6 \\
(497) \\
\end{array}$ \\
\hline $\begin{array}{l}\text { Family Medicine } \\
\text { (n) }\end{array}$ & $\begin{array}{c}133.9 \pm 61.9 \\
(5782) \\
\end{array}$ & $\begin{array}{c}78.1 \pm 42.4 \\
(189) \\
\end{array}$ & $\begin{array}{c}70.2 \pm 50.0 \\
(494) \\
\end{array}$ & $\begin{array}{c}66.9 \pm 47.6 \\
(789) \\
\end{array}$ & $\begin{array}{c}104.0 \pm 58.4 \\
(3099) \\
\end{array}$ \\
\hline $\begin{array}{l}\text { Total } \\
\text { (n) }\end{array}$ & $\begin{array}{c}111.7 \pm 65.3 \\
(10330)\end{array}$ & $\begin{array}{c}62.8 \pm 41.2 \\
(387)\end{array}$ & $\begin{array}{c}62.6 \pm 47.8 \\
(685)\end{array}$ & $\begin{array}{c}55.7 \pm 44.7 \\
(1411)\end{array}$ & $\begin{array}{c}81.8 \pm 57.5 \\
(5797)\end{array}$ \\
\hline
\end{tabular}

*excluding general internal medicine; includes cardiology, gastroenterology, hematology, respirology/resp medicine, rheumatology, immunology/allergy, neurology, micro/ID, nephrology, ID, medical oncology, endo/metabolism.

**includes general surgery, CV/T surgery, neurosurgery, orthopedic surgery, plastic surgery, urology, ophthalmology, otolaryngology, and ob/gyn

TABLE 2. Influence of physician age. Patient visits per week (mean \pm SD) and income source

\begin{tabular}{|c|c|c|c|c|c|}
\hline Age & $\geq 90 \%$ FFS & $\geq 90 \%$ Contract & $\geq 90 \%$ Sessional & $\geq 90 \%$ Salary & Not $\geq 90 \%$ of any source \\
\hline $\begin{array}{l}<35 \mathrm{yr} \\
\text { (n) }\end{array}$ & $\begin{array}{c}66.3 \pm 41.9 \\
(219)\end{array}$ & & & $\begin{array}{c}42.6 \pm 35.6 \\
(38)\end{array}$ & $\begin{array}{c}52.8 \pm 41.3 \\
(174)\end{array}$ \\
\hline $\begin{array}{l}35-44 \mathrm{yr} \\
(\mathrm{n})\end{array}$ & $\begin{array}{c}84.1 \pm 56.2 \\
(1011) \\
\end{array}$ & $\begin{array}{c}45.5 \pm 28.7 \\
(47)\end{array}$ & $\begin{array}{c}46.6 \pm 36.4 \\
(45)\end{array}$ & $\begin{array}{c}43.4 \pm 37.1 \\
(179) \\
\end{array}$ & $\begin{array}{c}54.1 \pm 38.2 \\
(666) \\
\end{array}$ \\
\hline $\begin{array}{l}45-54 \mathrm{yr} \\
(\mathrm{n})\end{array}$ & $\begin{array}{c}87.2 \pm 59.7 \\
(1343)\end{array}$ & $\begin{array}{c}50.4 \pm 35.7 \\
(66)\end{array}$ & $\begin{array}{c}42.1 \pm 37.6 \\
(48)\end{array}$ & $\begin{array}{c}44.5 \pm 39.7 \\
(182)\end{array}$ & $\begin{array}{c}59.9 \pm 48.4 \\
(936)\end{array}$ \\
\hline $\begin{array}{l}55-64 \mathrm{yr} \\
(\mathrm{n})\end{array}$ & $\begin{array}{c}87.7 \pm 59.3 \\
(1164)\end{array}$ & $\begin{array}{c}46.8 \pm 28.1 \\
(47)\end{array}$ & $\begin{array}{c}35.5 \pm 24.1 \\
(36)\end{array}$ & $\begin{array}{c}35.4 \pm 30.9 \\
(162)\end{array}$ & $\begin{array}{c}57.1 \pm 44.0 \\
(618)\end{array}$ \\
\hline $\begin{array}{l}\geq 65 \mathrm{yr} \\
\text { (n) }\end{array}$ & $\begin{array}{c}71.4 \pm 56.8 \\
(727)\end{array}$ & & $\begin{array}{c}31.6 \pm 21.4 \\
(49)\end{array}$ & $\begin{array}{c}41.1 \pm 30.3 \\
(39)\end{array}$ & $\begin{array}{c}44.2 \pm 37.6 \\
(255)\end{array}$ \\
\hline
\end{tabular}

Data reproduced with permission, from CMA/RCPSC/CFPC. CMA/RCPSC/CFPC own the copyright to the data. 